\title{
Separation of the thermal and optical properties and error limits in thermal depth profiling of fibre- reinforced materials
}

\author{
by J. Bolte', K. Simon ${ }^{2}$ and J. Pelzl $\left.\right|^{1}$ \\ ${ }^{1}$ Institut für Exp.-phys. III, AG Festkörpersp., Ruhr-Universität Bochum, D-44780 Bochum, FR Germany \\ ${ }^{2}$ WIS Munster, D-29623, FR Germany
}

\begin{abstract}
In thermal depth profiling of fibre-reinforced materials, the thermal and the optical properties were determined separately and with low relative errors. This is mainly due to the fact that the thermal and optical properties of the measured samples affect the measured photothermal phase at different modulation frequencies corresponding to different characteristic length of the thermal diffusion and optical absorption. The error limits of the thermal and optical parameters are determined by the root-mean-sqare deviations of the measured phases and by the error of the measurement of the static sample temperature.
\end{abstract}

\section{Introduction}

The thermal depth profiles of fibre-reinforced materials were measured perpendicularly to the main fibre direction by recording the radiometric signal as a function of the modulation frequency of thermal waves. The experimental setup is schematically shown in figure 1 . The thermal waves were excited by using a modulated $\mathrm{Ar}^{+}$laser beam, and the thermal wave response was detected with the help of a photoconductive $\mathrm{HgCdTe}$ detector and an IR optical system. The detected area was about $2 \mathrm{~mm}^{2}$. Amplitudes and phases of the thermal waves were measured over a frequency interval from $0.1 \mathrm{~Hz}$ to $80 \mathrm{kHz}$.

\section{Noise measurements}

In order to discuss the error limits of detection and interpretation, the errors of the measured amplitudes and phases were measured for each adjusted modulation frequency. This has been done by measuring a large number of values of the amplitude and phase within a time interval of about 50 times the lock-in integration time. From these data the average value and the rootmean-square deviations of the amplitude and phase were computed and recorded.

Figure 2 shows the root-mean-square deviations of the measured phases of a sample (T800) and a reference material (GG) plotted versus the modulation frequency. The reference material is a sample of smooth, homogeneous glassy carbon (Sigradur). One can see that the deviations of the phases increase with increasing frequency. This is due to the fact that the amplitudes of thermal waves strongly decrease with increasing frequency.

In Figure 3 the normalized phases of the sample T800 are plotted versus the square root of the modulation frequency. The error bars for the presented normalized phases are obtained from the errors of the two signals, sample and reference, by using the Gaussian law of error,

$$
\Delta \varphi_{n}=\sqrt{\left(\Delta \varphi_{s}\right)^{2}+\left(\Delta \varphi_{r}\right)^{2}}
$$

where $\Delta \varphi_{n}, \Delta \varphi_{s}$ and $\Delta \varphi_{r}$ are the root-mean-square deviations of the normalized phases and of the measured phases of the sample and reference, respectively.

\section{Texture effects}

Scanning electron micrographs revealed, that the sample T800 has got a thin cover layer of woven fibres which shows soft regular textures with characteristic depths of about $10 \mu \mathrm{m}$ and 
characteristic lateral extensions of $200-250 \mu \mathrm{m}$, contributing to a regular variation of the cover layer thickness within the detected surface area. It can be seen, that the cover layer of the sample T800 mainly consists of two different thicknesses, namly $2.3 \mu \mathrm{m}$ and $12 \mu \mathrm{m}$. Consequently, a superposition of different thermal response signals, corresponding to a laterally changing layer thickness of the thermally thin first layer, can be expected for the detection spot area. In locally resolved photothermal imaging, this lateral variation of the photothermal front surface signal has also been measured [1].

In general, the measured signal is proportional to the complex thermal wave, integrated over the focused spot area:

$$
\delta M(f, T) \propto \int_{A_{s}} \delta T(A, f) d A
$$

where $A_{s}$ is the focused spot area. If the bulk of the sample is assumed to be thermally homogeneous, the sample can be described by a two-layer model with two different thicknesses of the first layer, as shown schematically in figure 4. If the effects of lateral heat propagation are reduced by a heating spot area larger than the detected surface area, the photothermal signal arising in the detector is a composite of two different signals $\delta M_{a}(f, T)$ and $\delta M_{b}(f, T)$, which are emitted from the two different regions of the sample surface of different thicknesses of the first layer.

$$
\delta M(f, T)=\delta M_{a}(f, T)+\delta M_{b}(f, T) \propto \frac{A_{a}}{A_{s}} \delta T_{a}(f)+\frac{A_{b}}{A_{s}} \delta T_{b}(f)
$$

If the thermophysical and the optical properties of the considered material are homogeneous within the two layers, the measurement signal can be described by:

$$
\begin{aligned}
\delta M(f, T) \propto \frac{\eta I_{0}}{2 k_{1} \sigma_{1}} \frac{e^{i \omega t}}{\left(1-\frac{\sigma_{1}^{2}}{\beta_{1}^{2}}\right)}\left\{\frac{A_{a}}{A_{s}}\left[\frac{1+R_{12} e^{-2 \sigma_{1} l_{1 a}}}{1-R_{12} e^{-2 \sigma_{1} l_{1}}}-\frac{\sigma_{1}}{\beta_{1}}\right]\right. \\
\left.+\frac{A_{b}}{A_{s}}\left[\frac{1+R_{12} e^{-2 \sigma_{1} l_{1 b}}}{1-R_{12} e^{-2 \sigma_{1} l_{1} b}}-\frac{\sigma_{1}}{\beta_{1}}\right]\right\}
\end{aligned}
$$

where $R_{12}$ is the thermal reflection coefficient.

Figure 5 shows the theoretical approximation of the normalized phases of the sample T800. The two dashed lines in figure 5 are 2-layer solutions, with identical thermal and optical parameters but with a different thickness of the first layer $\left(l_{1 a}=2.3 \mu \mathrm{m}\right.$ and $\left.l_{1 b}=12 \mu \mathrm{m}\right)$, and the solid curve is the vectorial superposition of the two signals, calculated with the help of equation (4). It is remarkable, that a more detailed geometrical description of the cover layer and thus a superposition of more than two different photothermal signals leads to the same thermal and optical parameters.

\section{Separation of thermal and optical properties}

The phase profile at lower frequencies is determined by the thermal properties of the considered sample, namely the ratio of the effusivities $e_{s} / e_{b}$ of the first and second layer and the thermal diffusivity $\alpha_{s}$ of the first layer. This is shown, in agreement with Bennett and Patty [2], by theoretical simulations, presented in figure 6 by variations of the ratio of the effusivities $g_{12}=e_{s} / e_{b}$. Distinct values of the theoretical curves can only be seen at intermediate 
frequencies, namely in the region of the absolute phase minimum. In this case it is remarkable that the phases at high frequencies remain completely unchanged.

On the other hand, the high frequency limit of the phases, $\varphi_{n}(f \rightarrow 80 \mathrm{kHz})$, is mainly determined by the optical absorption constant $\beta_{1}$ of the cover layer. This is shown by variations of the optical absorption constant $\beta_{1}$ presented in figure 7 . The phase profile changes only at higher frequencies (in this case $f>2.5 \mathrm{kHz}$ ), while the phase and the frequency value of the absolute minimum of the theoretical curves $\left(\varphi_{\mathrm{n}} \approx 24^{\circ}, f \approx 1 \mathrm{kHz}\right)$ astonishingly remain completely unchanged. For higher values of the absorption constant, $\beta_{1} \rightarrow \infty$, the normalized phases at high frequencies, $\varphi_{n}(f \rightarrow 80 \mathrm{kHz})$, tend to zero and for lower values the phases take on almost constant negative values. Consequently the absorption constant can be determined with relatively good accuracy from the high frequency limit of the measured normalized phases.

\section{Error limits}

Based on the error limits measured for the different frequency regions (figure 3), the relative errors of the thermal and optical properties have been determined with the help of simulations as presented in figure 8 and figure 9 . As can be seen in figure 9, the optical absorption constant $\beta_{1}$ can be determined within a relative error of $13 \%$. For the ratio of the effusivities the relative error can be estimated to be $1 \%$. This very low error is due to the low limit of error measured for the phases at intermediate frequencies. In an analogous way the thermal diffusivity can be determined within a relative error of $5 \%$.

For the determination of the absolute value of the effusivitiy of the first layer $\mathbf{e}_{s}$ the normalized amplitudes have to be considered. For highly absorbing and thermally insulating samples, like the measured fibre-reinforced materials, the signal amplitude does not only depend on the amplitude of the thermal wave, but also on the static temperature rise during the measurement process [3]. The complex radiometric signal $\delta M(f, T)$ of the thermal wave can be described by

$$
\begin{aligned}
\delta M(f, T) & =4 C g(f) \varepsilon \sigma_{S B} T^{3}\left[\gamma(T)+\frac{T}{4} \frac{\partial \gamma(T)}{\partial T}\right] \delta T(f) \\
& =4 C g(f) \varepsilon \sigma_{S B} T^{3} \Gamma(T) \delta T(f)
\end{aligned}
$$

where $C$ is a constant, $g(f)$ describes the frequency dependence of the measurement setup, $\varepsilon$ is the emissivity of the sample and $\sigma_{S B}$ is the Stefan-Boltzman constant. $\gamma(T)$ is the effectivity with which the detection system is able to detect the IR radiation from a sample of static temperature $T$, and $\Gamma(T)$ describes the effectivity, with which the detection system is able to detect the IR radiation of a thermal wave at the static temperature $T$ [4].

In the case of a homogeneous sample and one-dimensional heat propagation, the normalized amplitudes $S_{n}$ can be described by [4]:

$$
S_{n} \propto \frac{T_{s}^{3}}{T_{r}^{3}} \frac{\Gamma\left(T_{s}\right)}{\Gamma\left(T_{r}\right)} \frac{e_{r}}{e_{s}}
$$

where the indexes ${ }_{n} s^{\prime \prime}$ and ${ }_{n} r$ mark the sample and the reference material, respectively. $T$ is the static temperature at the detected spot. The main error in determining the effusivity $e_{S}$ is caused by the error of the measurement of the static temperature $T$, which we determined to be about 2 $\mathrm{K}$ in our measurements. Consequently the relative error in determining the effusivity $e_{S}$ is about $4 \%$. Owing to the fact, that the thermal transport parameters of the first layer of the measured samples are higher than those of the layers deeper below the surface, reliable values for effusivity can only be obtained, if the effects of lateral heat propagation are reduced. Here, this has been achieved by a heating spot area, which is large in comparison to the detected surface area. 


\section{Conclusions}

From theoretical approximations and variations for the various physical parameters, we conclude that the thermal properties of the measured samples can be determined at intermediate frequencies, whereas the optical properties can be determined in the high frequency limit. This is possible due to the fact that the thermal and optical properties of the measured materials affect the photothermal signal on different length scales. The measured error limits, recorded as root-mean-square deviations of the measured phases, increase with the modulation frequency, from about $0.15^{\circ}$ at intermediate modulation frequencies to $1.2^{\circ}$ at high frequencies. Based on the simulation curves, the error limits measured for the different frequency regions are interpreted as error limits of the physical properties, i.e. the thermal diffusivity and effusivity and the optical absorption constant.

\section{Acknowledgments}

In part, this work has been supported by the Federal Ministry of Defence.

\section{REFERENCES}

[1] BOLTE (J.) .- Texture effects in thermal depth profiling of fibre-reinforced materials. To be publ. 1996.

[2] BENNETT (C.A.) and PATTY (R.R.). - Thermal wave interferometry: a potential application of the photoacoustic effect. Appl. Opt. 21, 1982, p. 49.

[3] KANSTAD (S.O.) and NORDAL (P. E.). - Experimental aspects of photothermal radiometry. Can. J. Phys. 64, 1986, p. 1155.

[4] BOLTE (J.). - Untersuchung der thermischen Eigenschaften von faser-verstärkten Verbundmaterialien mittels frequenzabhängiger photothermischer Radiometrie. Diploma thesis, Ruhr-Universität Bochum, FRG, 1995. [in GERMAN]

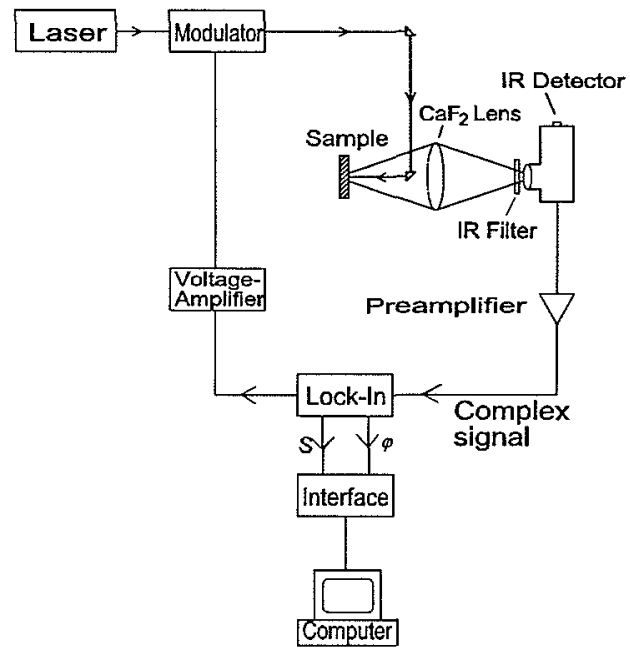

Fig. 1: Experimental setup 
$\mathrm{f} / \mathrm{KHz}$

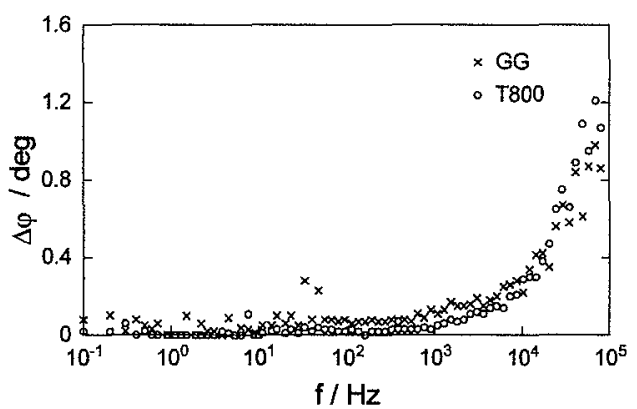

Fig. 2: Root-mean-sqare deviations of the measured phases

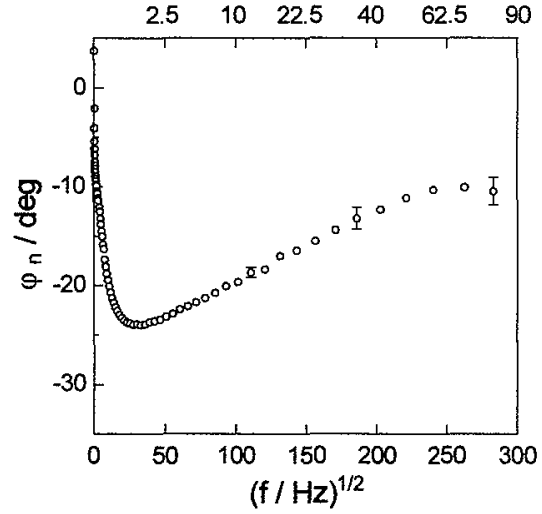

Fig. 3: Normalized phases of the sample T800 with error bars

$\mathrm{f} / \mathrm{KHz}$
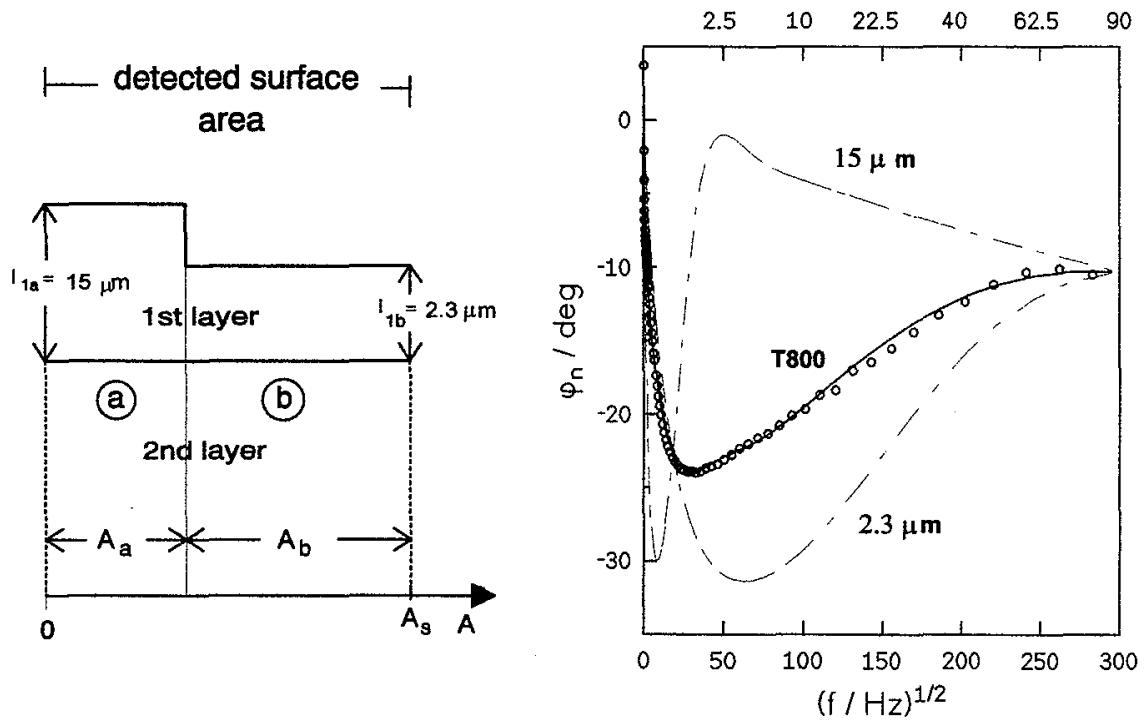

Fig. 4: Geometrical model of the sample T800

Fig. 5: Vectorial superposition of two thermal wave solutions in comparison with the measured phases obtained from a composite fibre reinforced sample 


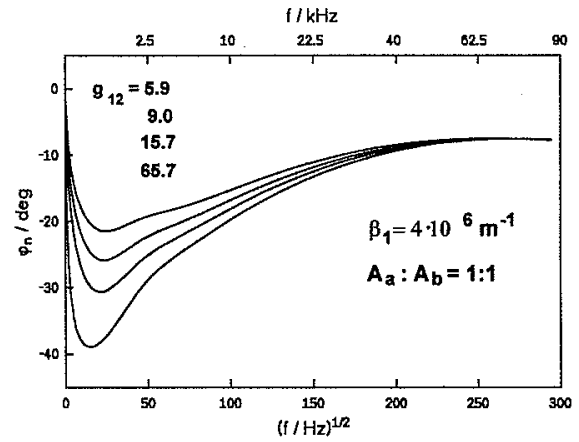

Fig. 6: Simulation of phase profiles: With a variation of the ratio of the effusivities $g_{12}=\theta_{\mathrm{s}} / e_{\mathrm{b}}$, the value of the relative minimum changes

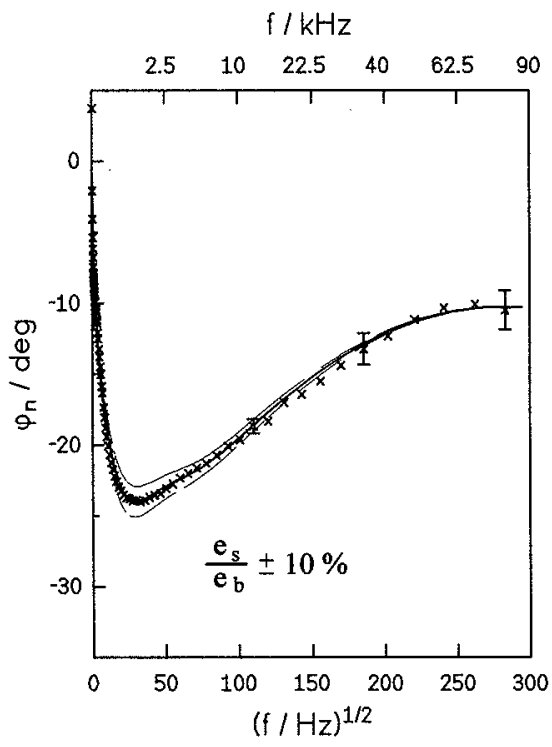

Fig. 8: Normalized phases in comparison with the optimal approximation and variations of the ratio of the effusivities (dashed lines)

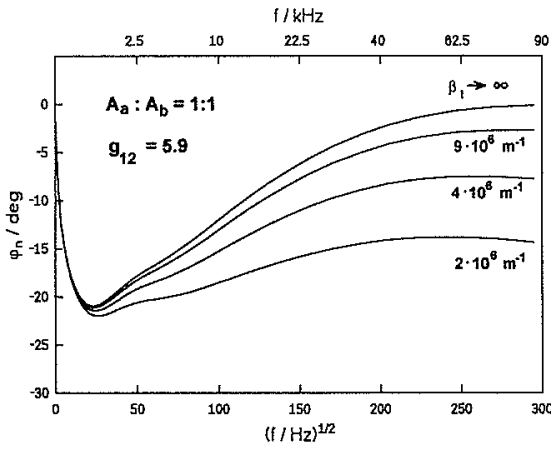

Fig 7: Simulation of phase profiles: With a variation of the optical absorption $\beta_{1}$, the high-frequency limit of the phase profile changes considerably

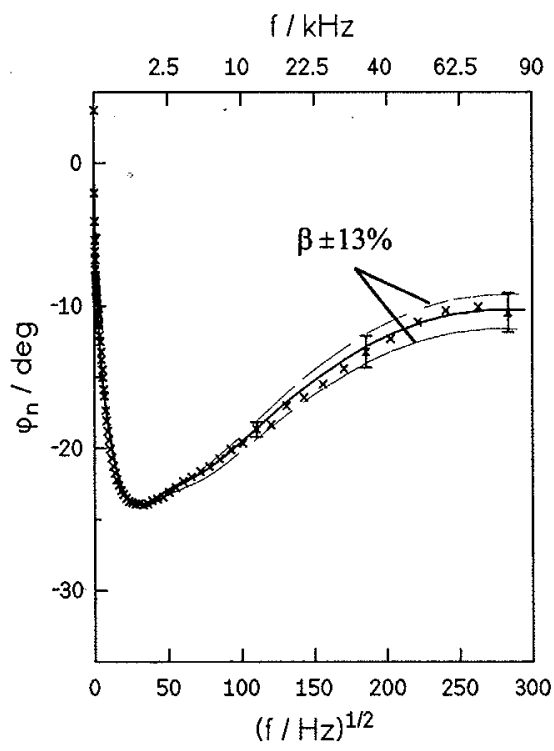

Fig. 9: Normalized phases in comparison with the optimal approximation and variations of the optical absorption constant (dashed lines) 